\title{
Fully Automated Pollen Analysis and Counting: The Pollen Monitor BAA500
}

\author{
U. Heimann ${ }^{1}$, J. Haus ${ }^{1}$, D. Zühlke ${ }^{2}$
}

Helmut Hund GmbH, Wilhelm-Will-Str. 7, 35580 Wetzlar-Nauborn

2 Fraunhofer-Institut für Angewandte Informationstechnik, Schloß Birlinghoven, 53754 Sankt Augustin

\section{Introduction}

Pollen allergies have become a wide-spread disease. Every year, during the bloom of many plants, about 12 million citizens of the Federal Republic of Germany (every seventh person!) suffer from acute symptoms that reach from allergic rhinitis to life-threatening anaphylactic shocks. For their daily routine, allergic persons strongly depend on up-to-date information on pollen counts in their area, e. g. to adjust their daily medications.

These allergies are caused by airborne pollen, and the state-of-the-art technique for counting the various pollen taxa is the so-called Burkhard trap. These traps suck ambient air onto a piece of adhesive tape where pollen and other aerosole particles are deposited. This tape will eventually be analyzed by human operators under a light microscope. This kind of analysis requires a high degree of experience and concentration, which - due to the ubiquitous human factor - cannot always be guaranteed. Some pollen taxa differ from their sizes alone, which cover a range between about $10 \mu \mathrm{m}$ to $150 \mu \mathrm{m}$, others can only be discriminated by judging minute details. As this is a time-consuming analysis, the daily pollen count will thus rely on data from the previous day.

\section{Innovation and Technical Solution}

This work describes the pollen monitoring system BAA500, realized by the Helmut Hund GmbH, Wetzlar, in cooperation with the Fraunhofer Institute for Applied Information Technology (FIT), Sankt Augustin, and with the Fraunhofer Institute for Toxicology and Experimental Medicine (ITEM), Hannover (Fig. 1).

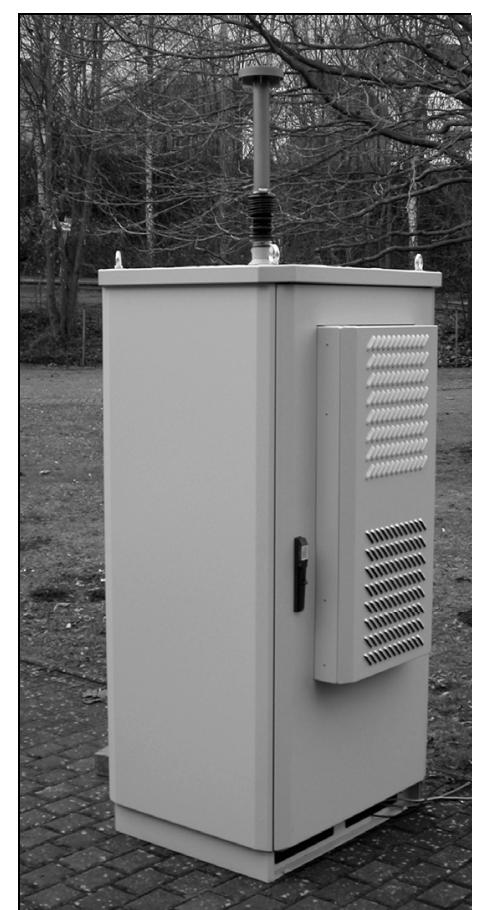

Fig. 1: Hund pollen monitoring system BAA500. 
Equipped with an automated pollen deposition and handling system and with an automated microscope in combination with an image analysis system, the BAA500 is capable of determining and counting six allergologically relevant pollen taxa and seven taxa that have no allergological impact (Tab. 1):

Tab. 1: Pollen taxa determined and counted by the pollen monitoring system.

\begin{tabular}{cc}
\hline Allergologically relevant & Allergologically not relevant \\
\hline Hazel (Corylus) & Maple (Acer) \\
Alder (Alnus) & Beech (Fagus) \\
Birch (Betula) & Yew (Taxus) \\
Grass without Rye (Poaceae) & Oak (Quercus) \\
Mugwort (Artemisia) & Hornbeam (Carpinus) \\
Ragweed (Ambrosia) & Rye (Secale) \\
& Willow (Salix) \\
\hline
\end{tabular}

The aerosoles are sampled with a three-stage virtual impactor. Its aerodynamic design ensures that all particles with aerodynamical diameters of more than $11 \mu \mathrm{m}$ are deposited on a specimen carrier. This carrier is equipped with a special substrate into which the aerosole particles sink after a subsequent heating step. Then, the sample is ready for analysis by an automated 3D-microscope.

The microscopic image is taken with a semiplan-achromatic objective SPL 20/0.50 (Hund) and with a $1 / 2$ " CCD camera with a resolution of 1.2 megapixels. The sample is illuminated with an LED with $530 \mathrm{~nm}$ wavelength and a microscope condenser with NA 0.9. With the scanning stage, stacks of 70 images with a z-distance of $1.5 \mu \mathrm{m}$ are recorded. For a full coverage of the sampling area, 350 of these stacks have to be recorded.

In the first image analysis step, the data of each image stack are reduced by optimizing the contrast across all images and combining the result in one synthetic 2D-image that contains all layers of maximum sharpness of the single images. Figure 2 shows an example for a single image in the plane of maximum sharpness, and Fig. 3 the corresponding synthetic image with the background deleted. From these synthetic images, all aerosole particles (objects) are extracted in a two-step segmentation process. Afterwards, the classification algorithm calculates different form and texture features. Based on these feature vectors, the objects are classified by the trained classificator (Fig. 4).

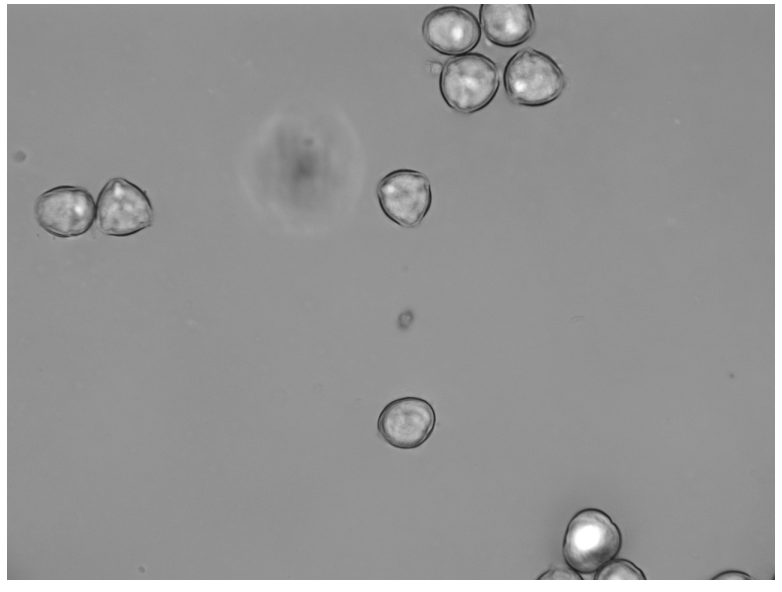

Fig. 2: Single stack image, plane of maximum sharpness.

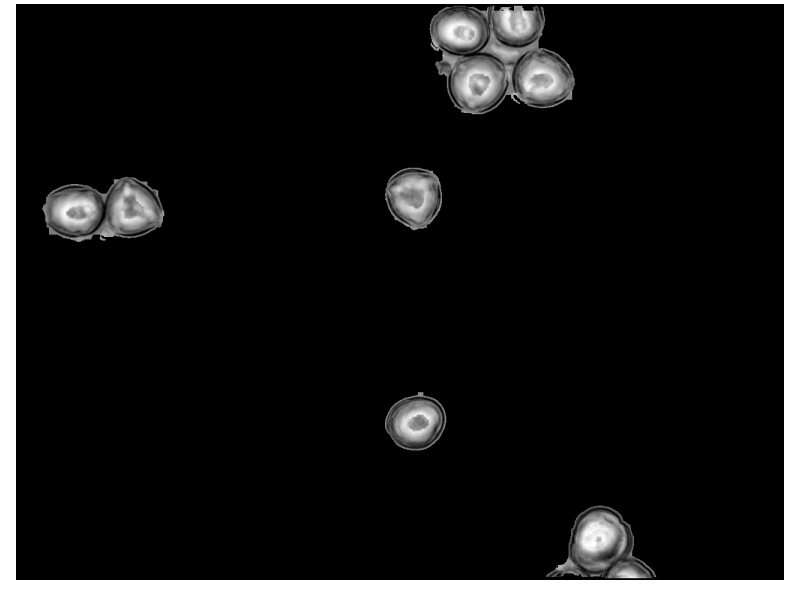

Fig. 3: Synthetic image. 


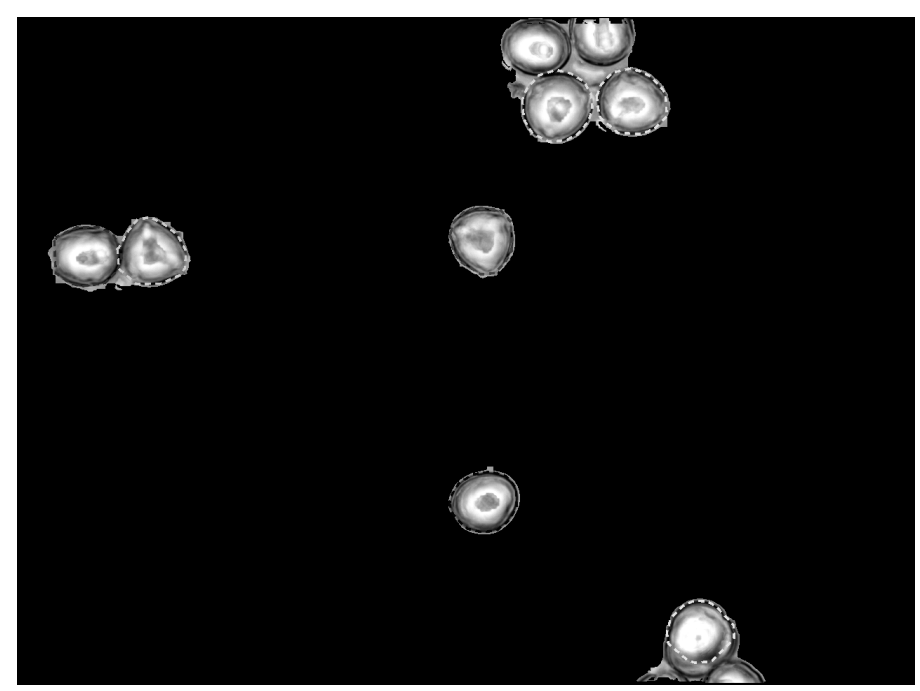

Fig. 4: Synthetic image with segmented and classified pollen (dashed contours).

The sampling intervals of the Pollen Monitor can be adjusted by the operator and can range from one to three hours. The device thus consumes between 8 and 24 sample carriers per day which are stored in a reusable magazine cassette. The total number of sample carriers guarantees autonomous operation over a time interval of two to four weeks. The two magazine cassettes that contain new and used sample carriers, respectively, can easily be changed by the operating personnel.

\section{Results}

The training of the classicator was done with natural pollen samples. Figures $2-4$ show typical images from these training procedures.

The results of the classification process are given in Tab. 2. The relevant statistical properties are:

$$
\begin{gathered}
\text { RECALL }=\frac{\text { Number }(\text { correctly classified pollen })}{\text { Number }(\text { pollen classified by human })} \\
\text { PRECISION }=\frac{\text { Number }(\text { correctly classified pollen })}{\text { Number }(\text { pollen classified by computer })}
\end{gathered}
$$

Tab. 2: Test results of classification process. Pollen taxa as given in Tab. 1.

\begin{tabular}{lcc}
\hline Pollen taxa & Recall (average) & Precision (average) \\
\hline Allergologically relevant & $94 \%$ & $92 \%$ \\
Allergologically not relevant & $96 \%$ & $94 \%$ \\
Non-pollen objects & $80 \%$ & $90 \%$ \\
\hline
\end{tabular}


To guarantee these results, however, quality assurance of the overall process from air intake to classification is mandatory. This is why the evaluation and the improvement of the quality of the entire analysis is an ongoing project.

\section{Discussion}

With the BAA500, the first fully-automated pollen counter has been introduced into the market. It is designed to operate autonomously over 24 hours a day and 7 days a week. Its supply of sample carriers allows continuous operation for a period of more than two weeks before new magazin cassettes have to be inserted.

The device is designed to work in a network of several pollen monitoring systems with which a spatially resolved pollen count will be possible. With the short time interval that it requires to yield a counting result, the BAA500 will now allow reliable pollen forecasts with a significantly higher frequency than with conventional methods. 